\title{
Engagement organisationnel en situation de changement: quelles perspectives dans un hôpital public? Revue de question
}

\author{
Elisabeth Doutre ${ }^{1}$ \\ Université Pierre Mendès France
}

\begin{abstract}
This study aims to identify which variables are presents in a context of organizational change and to examine how they are antecedents of the leader's organizational commitment (EO), either separately or jointly. These variables can also have some effects on the collaborator's affective commitment to the supervisor. The case of a French public hospital will be-taken as an example to develop the context of these perspectives of research and application.
\end{abstract}

Key words: Organizational commitment, affective commitment, commitment towards the supervisor, organizational change, public organization.

\section{Rezumat}

Acest studiu îşi propune identificarea variabilelor prezente într-un context de schimbare organizațională şi investigarea manierei în care acestea se constituie ca antecedente ale angajamentului organizațional al liderului, fie idependent unele de altele, fie în interacțiune. Aceste variabile pot de asemenea influența angajamentul afectiv al colaboratorului față de supervizor. Cazul unui spital public din Franța va fi luat ca şi exemplu pentru dezvoltarea contextului acestor perspective de cercetare şi aplicații.

Cuvinte cheie: angajament organizațional, angajament afectiv, angajament față de superior, schimbare organizațională, organizație publică.

Obtenir l'engagement des salariés pourrait-il être aujourd'hui considéré comme l'une des conditions de réussite des changements introduits dans l'organisation de travail? Certains auteurs le laisseraient penser (Fisch, Weakland \& Segal, 1982; Hersey \& Blanchard, 1987; Fiedler, 1986; Bandura, 2001; Brasseur \& Mzabi, 2003, Paillé, 2006). S'il apparaît évident que sans les acteurs organisationnels, aucune pratique n'est envisageable, certaines formes d'engagement ne pourraient - elles pas favoriser la concrétisation de projets de changement, et auquel cas, quel modèle permettrait-il le mieux de le faire?

Notre objectif ici est de réaliser un état de l'art pour comprendre comment s'organisent et se structurent les mécanismes d'engagement organisationnel. Quelles sont les diverses variables, comment s'articulent leurs effets lorsqu'elles jouent conjointement sur l'engagement organisationnel du chef et de ses collaborateurs dans une démarche de changement, notamment dans un contexte organisationnel de service public. Ce dernier a fait l'objet de nombreuses études par différents experts. Ces études montrent la difficulté d'obtenir cet engagement en situation de modernisation organisationnelle notamment à l'hôpital. Certains auteurs expliquent cela par une inertie bureaucratique (Warin, 2004), d'autres par une inadaptation des méthodes utilisées (François, 2003; Meyer, \& al, 2002).

Nous tenterons de clarifier le concept d'engagement dans un premier temps, celui de changement ensuite et enfin nous tenterons une analyse explicative des difficultés rencontrées à ce sujet dans le cadre de l'introduction d'un changement tel que la modernisation d'un service public.

\footnotetext{
${ }^{1}$ Adresa de corespondență:

Elisabeth.doutre@upmf-grenoble.fr
} 


\section{L'engagement: un concept multi objet et multiforme}

Si l'engagement au travail a suscité de nombreux travaux de recherche, sa clarification se heurte à la coexistence de multiples approches. Morrow (1983) a ainsi recensé plus de trente définitions différentes de ce concept dans la littérature anglosaxonne à travers le terme de « commitment ». Deux courants cependant prédominent la recherche, celui de l'engagement comportemental lié aux travaux de Festinger (1957) et se traduisant par une implication dans les actes, un "passage aux actes » selon Joulé \& Beauvois (1984) en étant au cœur de la théorie de la rationalisation (Beauvois \& Joulé, 1985), et celui de l'engagement attitudinal. $\mathrm{Ce}$ dernier, plus connu sous le terme d'engagement organisationnel, est associé à une relation entre deux entités, un sujet, et une composante de la situation de travail, un objet. Cet engagement organisationnel «part du principe que l'on ne peut être membre d'un groupe sans construire progressivement avec lui une relation et sans être construit en partie soi-même dans cette relation 》 (Thévenet, 1993, p.33).

\section{L'engagement attitudinal}

Dans ce courant théorique, ce rapport "sujet-objet » est toujours défini comme une attitude (Lincoln \& Kalleberg, 1996). Trois points essentiels concernant l'attitude permettent de définir les approches sur l'engagement organisationnel: l'attitude est interne au sujet, elle a un objet et elle comporte trois dimensions (Brasseur \& Mzabi, 2003).

L'attitude est « interne»: que ce soit un mécanisme psychologique, un état mental ou une tendance psychologique (Eagly \& Chaiken, 1993), l'attitude est un processus qu'il est impossible d'observer directement «puisque interne au sujet » (Tafani \& Souchet, 2001, p.59). Cet engagement des sujets n'est alors jamais vraiment acquis car il n'est identifiable que par son expression ou son extériorisation: il y a là un lien, certes complexe mais réel, entre attitude et comportement. Nos actes peuvent construire ou modifier nos attitudes (Beauvois \& Joulé, $1985)$ et ne relèvent pas du même indicateur mais ce rapport de cause à effet peut être aussi inversé: des travaux attribuant à l'engagement organisationnel l'absentéisme, le turn over ou la performance considèrent le comportement comme la résultante de l'attitude (Wierner \& Vardi, 1980; Mathieu \& Zajac, 1990; Blau, 1999; Cohen, 2000). Nos attitudes peuvent donc transformer nos comportements (Brown, 1996);

L'attitude a un "objet»: si les attitudes spécifiques à un contexte ont toujours un objet, cela signifierait aussi qu'un sujet ne serait jamais « pour » dans l'absolu (Fisbein \& Ajzen, 1975). Par exemple, le sujet pourra adhérer à la finalité d'un projet donné, mais il pourra être opposé à ses modalités de mise en œuvre. Son adhésion ponctuelle ne pourra pas, par ailleurs, s'appliquer systématiquement à tous les projets futurs. Les recherches les plus développées et les plus fréquentes sont celles qui abordent le rapport entre le sujet et sa situation de travail par le biais de son attitude vis-à-vis de son organisation, de ses valeurs, de ses buts ou encore de son système hiérarchique. Cet engagement organisationnel pourra être compromis par des conflits identitaires ou par des relations discordantes dans le groupe de travail (Angle \& Perry, 1983; Reichers, 1985; Yoon, Baker \& Ko, 1994).

Enfin, l'attitude présente « trois dimensions»: une dimension cognitive, associée à la pensée, au jugement ou encore aux croyances du sujet, une dimension affective, qui renvoie à ses émotions et sentiments, et une dimension conative, qui regroupe les intentions d'action (Rosenberg \& Hovland, 1960). Ces trois dimensions permettent de différencier les processus internes à l'œuvre dans l'engagement organisationnel.

\section{L'engagement organisationnel (EO)}

Les recherches sur l'Engagement Organisationnel sont centrées essentiellement sur ces trois dimensions.

Les premières études se sont tout d'abord situées sur la dimension affective et ont pris essentiellement en compte l'attachement affectif, émotionnel du salarié (Mowday, Porter, \& Steers, 1982): c'est une relation entretenue entre les membres de l'équipe, avec un partage des objectifs sous l'angle de l'attachement affectif. Cet attachement développe une identification à son travail et une identification aux valeurs de l'organisation. Relié au sentiment d'appartenance, l'Engagement Affectif (EA) reste toujours défini comme un attachement 
psychologique ressenti par le sujet (Lincoln \& Kalleberg, 1996; Cohen, 2000; Meyer \& Herscovitch, 2001). La dimension affective a été la plus étudiée, car elle semble être la plus prédictrice de comportements.

Concernant la dimension cognitive, les chercheurs vont définir ce type d'engagement comme une dynamique rationnelle interne aux sujets, parfois qualifiée d'Engagement Calculé (EC) (Commeiras, 1994). Inspirés par la théorie des avantages comparatifs (Becker, 1960), ces chercheurs considèrent que les salariés procèdent à une évaluation des bénéfices et des coûts professionnels et personnels de leur implication. Kanter (1968) introduit le concept d'engagement de continuité («continuance commitment») associé à la décision de partir ou de rester dans l'organisation; l'engagement du sujet est alors fonction de ce qu'il considère percevoir mais aussi de ses attentes vis-à-vis de sa situation professionnelle voire même de ses idéaux. Gaertner et Nollen (1992) montrent que les employés, qui croient qu'il existe des opportunités de carrière et qui pensent que l'entreprise essaie de garantir la sécurité de l'emploi, sont plus engagés dans l'entreprise que ceux qui ne le croient pas. Des recherches prennent en compte à la fois la dimension affective et la dimension cognitive et montrent le lien avec l'éthique du sujet (Morrow, 1983; O'Reilly \& Chatman, 1986): la conviction que le travail est un bien en soi, ou que la valeur morale se mesure aux efforts que l'on y fournit. Cette forme d'engagement fait intervenir soit la raison soit les sentiments ou les émotions dans une complexe articulation. Ainsi, l'individu pourra se contraindre de travailler en dépit de ce qu'il ressent, ou à l'opposé, éprouver de la joie en concordance avec ses valeurs et ses croyances.

Enfin, l'Engagement Normatif (EN) fait référence à l'ensemble des pressions normatives internalisées qui poussent un individu à agir dans le sens des objectifs et des intérêts de l'organisation, non pas pour en retirer un bénéfice, mais parce qu'il est «normal » d'agir ainsi (Wiener, 1980; Meyer \& allen, 1997). Cette dimension contribuerait au renforcement du sentiment d'identité ou encore du sentiment d'appartenance.

Des approches tridimentionnelles vont définir l'engagement organisationnel comme un état psychologique résultant d'un processus complexe d'articulation et de priorisation (Allen \& Meyer, 1991, 1996). Ce processus résulterait à la fois des désirs, de la volonté, des devoirs, des intérêts du sujet, des intentions d'action dans l'organisation ou/et des intentions de quitter l'entreprise. Ces auteurs proposent de définir l'engagement organisationnel comme une attitude de l'individu correspondant d'une part à son attachement affectif à l'organisation dans laquelle il travaille, et au partage des valeurs communes, et d'autre part, à son choix raisonné de lui rester fidèle en fonction des coûts associés à son départ, et enfin, à l'obligation morale qu'il ressent d'accomplir son devoir (Varona, 1996; Meyer \& al, 2001; Durrieu \& Roussel, 2002). C'est un état qui traduit finalement tout autant l'attitude que le comportement (Mathieu \& Zajac, 1990, Herrbach \& Mignonac, 2002; Meyer \& al, 2002). Tous les auteurs semblent s'accorder sur ces concepts de base.

\section{Des mécanismes efficaces}

L'identification (la relation gratifiante avec l'organisation) et l'internalisation (congruence entre valeurs du sujet et objectifs de l'organisation) peuvent être considérés comme les meilleurs mécanismes par lequel l'Engagement Affectif (EA) se développe. Contrairement à l'Engagement calculé, l'EA oriente positivement les comportements individuels à l'égard de l'organisation (Meyer \& al, 2002). Cependant, ces composantes attitudinales pourraient diverger: le sujet en désaccord avec les valeurs de son organisation ne partira pas nécessairement car ce n'est pas son intérêt ou parce qu'il ne pense pas pouvoir trouver un autre travail dans une autre organisation, ou encore parce qu'il est fonctionnaire...Ce sujet sera alors engagé «par défaut» (Becker, 1960). Cette particularité de l'engagement calculé doit donc être prise en compte dans l'élaboration des programmes de changement dans les services publics.

\section{Les recherches sur l'Engagement Organisationnel}

Nombre de recherches se sont alors orientées soit sur les antécédents de l'engagement organisationnel (Allen \& Meyer, 1990; Simard, 2000), soit sur ses conséquences sur le comportement des sujets (Mathieu \& Zajac, 1990; Palmero, 2000), soit encore sur les deux à la fois (Meyer \& al 2002). 
Les recherche nombreuses sur les antécédents ont montré différents liens significatifs: le lien avec l'intention de départ a été étudié (Hartman \& Bambacas, 2000): les dimensions affective et calculée sont significatives dans leur relation avec l'intention de départ. L'EA serait aussi associé à une meilleure performance individuelle et collective (Meyer, Allen \& Smith, 1993), à la réduction des retards et de l'absentéisme (Mathieu \& Zajac, 1990). Les variables organisationnelles semblent plus prédictibles d'engagement organisationnel que les variables individuelles: la décentralisation des décisions, la délégation de responsabilités, l'autonomie au travail, le climat de soutien, semblent avoir un impact significatif dans le développement de l'engagement affectif (EA). Des perceptions négatives de la justice et l'iniquité des pratiques d'évaluation, de rémunération, et de promotion peuvent endommager la nature de l'engagement affectif et normatif des salariés envers l'organisation (Meyer \& Smith, 2000). Certaines études se sont intéressées à l'engagement organisationnel de sujets à hauts potentiels: le rôle des pratiques de développement des compétences, de participation à la décision et de rémunération individualisée semblent déterminants (Mannheim, Baruch \& Tal, 1997; May, Korczynski \& Frenkel, 2002) de même que la relation groupe/leader. Des études récentes à ce sujet (Paillé, 2006) ont vérifié le lien significatif de la pratique de la responsabilisation et de la gestion des carrières sur l'engagement affectif (EA), de la rémunération et des pratiques de recrutement sur l'engagement normatif (EN), et enfin des pratiques de formation et de responsabilisation sur l'engagement calculé (EC) ( El Akremi \& Trabelsi, 2003). Enfin, il a été démontré que les trois dimensions s'influencent entre elles, l'engagement affectif influence l'engagement normatif (Meyer \& Smith, 2000) qui lui-même semble influencer l'engagement calculé (Delobbe \& Vandenberghe, 2000).

L'évolution du concept d'EO vers l'engagement vis-à-vis du supérieur

Plus récemment, le modèle de l'EO a été appliqué au concept d'engagement vis-àvis du supérieur. Meyer et Herscovitch (2001) ont proposé une généralisation du modèle partant de l'idée que les trois dimensions de l'EO prenaient leurs racines dans des états mentaux différents. Selon eux, les états mentaux du désir (EA) d'obligation perçue $(E N)$ et de coût perçu (EC) pouvaient être le terreau d'engagement envers de nombreuses entités ou objets tel notamment le supérieur. Plusieurs recherches empiriques ont mis en évidence que les trois dimensions de l'EO pouvaient dépeindre un engagement « envers le supérieur ». Ce report d'engagement sur le supérieur résulterait d'une diminution de la relation identitaire entre l'employé et son organisation due notamment aux bouleversements économiques (Eisenberger \& al, 2002, Stinglhamber \& al, 2002). Dans ce cas là, l'engagement affectif envers le supérieur résulterait du sentiment d'identité partagée et d'adhésion à ses valeurs, du sentiment de loyauté à son égard et du sentiment de reconnaissance que pratiquerait le supérieur vis-à-vis des investissements personnels réalisés par l'employé. L'EN envers le supérieur se définirait comme la conséquence de bénéfices reçus du supérieur et l'EC envers le supérieur serait la conséquence psychologique des investissements réalisés par l'employé dans sa relation de travail avec son chef (Stinglhamber \& al, 2002, p.141).

A la lecture du tableau, on constate une influence largement démontrée des variables organisationnelles et managériales sur l'Engagement Affectif (EA). En effet, on peut compter 6 variables uniquement antécédentes de l'EA, et 6 autres variables antécédentes de l'EA et l'Engagement calculé ou de continuité (EC). On montre que ces variables organisationnelles sont prédictibles, notamment dans leur capacité explicative du degré d'engagement (Meyer \& Allen, 1997). II est donc possible de penser que l'influence des variables individuelles reste encore un champ à explorer notamment dans leurs liens avec ces variables organisationnelles.

La relation employé/leaders qui a été explorée dans le cadre de l'EO envers le supérieur peut s'expliquer par le désir de croire qu'une relation identitaire entre le collaborateur et son chef est essentielle au maintien d'une motivation extrinsèque des employés, notamment dans les grands groupes (De Cotiis \& Summers, 1987; Stinglhamber \& al, 2002).

Espérer voir se développer un engagement affectif envers le supérieur ne pourrait-il pas aussi laisser espérer une moindre résistance au changement des employés? En maintenant un engagement envers le supérieur, l'organisation ne pourraitelle pas alors compenser les pertes de repères que les changements génèrent? Ces pertes 
Tableau 1. Liens significatifs entre les différentes variables

\begin{tabular}{|c|c|c|}
\hline $\begin{array}{lll}\text { Variables antécédentes à l'engagement } \\
\text { organisationnel }\end{array}$ & $\begin{array}{l}\text { Type d'engagement: } \\
\text { E0: engagement } \\
\text { organisationnel } \\
\text { EA: engagement affectif } \\
\text { EC: engagement de } \\
\text { continuité ou calculé } \\
\text { EN: engagement } \\
\text { normatif }\end{array}$ & Variables dépendantes \\
\hline $\begin{array}{l}\text { Reconnaissance du supérieur } \text { immédiat } \\
\text { (Simard, 2000) } \\
\text { Reconnaissance du leader (De Cotiis \& } \\
\text { Summers, 1987) }\end{array}$ & EO & \\
\hline $\begin{array}{l}\text { Variables individuelles: } \\
\text { Ancienneté (Meyer \& al, 2002) } \\
\text { Auto valorisation des composantes identitaires } \\
\text { (Herrbach \& Mignonac, 2002) }\end{array}$ & EA & $\begin{array}{l}\text { Performance individuelle (Meyer \& al, 1993) } \\
\text { Performance collective (Meyer \& al, 1993) } \\
\text { Adoption de comportement de citoyenneté } \\
\text { organisationnelle (Meyer \& Allen, 1997) } \\
\text { Mobilisation (Simard, 2000) } \\
\text { Réduction des retards (Mathieu \& Zajac, } \\
\text { 1990) } \\
\text { Réduction de l'absentéisme (Mathieu \& Zajac, } \\
\text { 1990) } \\
\text { Réduction du turn over (Mathieu \& Zajac, } \\
\text { 1990) }\end{array}$ \\
\hline $\begin{array}{l}\text { Variables organisationnelles } \\
\text { Type d'organisation et dimension du pouvoir du } \\
\text { chef } \\
\text { (Meyer \& al, 2002) } \\
\text { Justice interactionnelle (Meyer \& a, 2002) } \\
\text { Justice distributive (Meyer \& al, 2002) } \\
\text { L'autonomie dans le travail (Matieu \& Zajac, } \\
\text { 1990; Simard, 2000) } \\
\text { Partage de l'information (Varona, 1996) }\end{array}$ & EA & \\
\hline $\begin{array}{l}\text { Le développement des compétences des } \\
\text { employés (Simard, 2000) } \\
\text { Les pratiques de formation (Brasseur \& al, } \\
\text { 2003) } \\
\text { La perception d'accès à la formation (Baling \& } \\
\text { al, 2000) } \\
\text { Les opportunités d'apprentissage au travail } \\
\text { (Meyer \& al, 2001) } \\
\text { La perception de justice (Meyer \& Smith, 2000) } \\
\text { La reconnaissance par le supérieur (Decotiis \& } \\
\text { Summers, 1987) }\end{array}$ & $E A+E C$ & \\
\hline $\begin{array}{l}\text { Partiques de rémunération (El Akremi \& } \\
\text { Trabelsi, 2003) } \\
\text { Les pratiques de recrutement (El Akremi \& } \\
\text { Trabelsi, 2003) } \\
\text { Dimension du pouvoir (Meyer \& al, 2002) } \\
\text { La justice interactionnelle (Meyer \& al, 2002) }\end{array}$ & EN & \\
\hline
\end{tabular}




\section{Le sentiment d'identité}

Il faut se connaître (concept de soi ou identité) avant de se reconnaître (estime de soi). En effet, tout individu doit connaître ses forces, ses habiletés, ses qualités, ses difficultés et ses limites personnelles pour qu'il puisse poser un jugement positif sur cette connaissance qu'il a de lui-même. Acquérir un sentiment d'identité positive est synonyme d'une bonne estime de soi car les repères sont stabilisés et donnent confiance en soi à l'individu. Le sentiment d'identité positive réfère à l'estime de soi globale. La connaissance de soi et l'identité personnelle qui en résultent, constituent les fondements de l'estime de soi. Ce sentiment d'identité est une des variables anticipatrices de l'Engagement Affectif (Herrbach \& Mignonac, 2002). Les définitions du concept d'identité doivent partir d'un modèle à "double entrée »: d'un côté il est nécessaire d'avoir la perspective individuelle (le soi) qui concerne la structure organisée de l'identité personnelle, et de l'autre la vision sociale qui se rattache à la culture et au sentiment d'appartenance qui fait référence à la structure organisée de l'identité collective.

Le sentiment d'identité se consolide si on augmente les relations avec le groupe. On voit bien les liens qui peuvent se faire entre l'identité, notamment l'identité sociale ou collective et l'engagement: l'identité est précisément le chemin de la sociabilité. L'ensemble des mécanismes relationnels qui soutiennent l'identité de soi d'un individu et sa projection sur une identité sociale est un des éléments fondamentaux des conduites humaines. L'identité sociale comprend les attributs catégoriels et statutaires qui se réfèrent à des catégories sociales où se rangent les individus (groupes, sous-groupes: « jeune », « femme », « cadre », « père »...). C'est souvent une identité «prescrite» ou assignée, dans la mesure où l'individu n'en fixe pas, ou pas totalement, les caractéristiques. Cette identité sociale situe l'individu à l'articulation entre le sociologique et le psychologique. Elle envisage, comme le souligne Tajfel et al (1999) le rôle joué par la catégorisation sociale qui selon lui « comprend les processus psychologiques qui tendent à ordonner l'environnement en termes de catégories: Groupes de personnes, d'objets, d'évènements [...] en tant qu'ils sont équivalents les uns aux autres pour l'action, les intentions ou les attitudes d'un individu» (p.165). Ce sentiment d'identité est donc une variable importante dont les effets sont à prendre en compte dans leur capacité à accompagner ou inhiber un engagement organisationnel. Et notamment en cas de changement organisationnel, dans lequel, des résistances attitudinales vont apparaître.

\section{Le changement organisationnel et l'engagement organisationnel: un lien d'intérêts communs}

Le changement organisationnel est une démarche aujourd'hui courante dans toutes les organisations de travail, et les diverses recherches faites à ce sujet montrent la complexité des mécanismes en jeu et les difficultés à contourner les résistances qui apparaissent (Cornet, 1999; Rondeau, 1999; 2002). Ces chercheurs montrent que les processus de transformation doivent s'ajuster continuellement aux contraintes de l'environnement. Dans cette perspective, un certain nombre de contraintes ont été explorées: la pression des groupes d'intérêt, le leadership des dirigeants, le type d'organisation du travail, et les ressources disponibles. Les différents travaux à ce sujet suggèrent l'existence d'étapes logiques par lesquelles passer afin d'obtenir des changements comportementaux gages des transformations attendues (Lewin, 1951; Judson, 1991; Kotter, 1996; Mintzberg, Ahlstrand \& Laampel, 1999). On parle alors d'un «processus » de changement. Des recherches récentes ont identifié des variables jugées les plus significatives dans le succès d'un processus transformationnel, ces variables permettraient de réduire notamment la résistance au changement et le délai de mise en œuvre des transformations (Rondeau, 1999; Paillé, 2006). Quatre familles de variables ont ainsi été constituées: des variables liées au contexte, d'autres liées au scénario de changement lui-même, celles liées aux acteurs, enfin, des variables liées à la progression du changement.

\section{Les variables contextuelles}

La complexité du changement est déterminante. Si le changement remet en cause la structure de l'organisation, les modes de gestion, les pratiques et les rôles, on est en face d'une situation complexe qui va nécessiter une véritable stratégie de mise en œuvre (un scénario) et ce d'autant plus si la 
légitimité de ces changements n'apparaît pas suffisamment dans le projet.

\section{Le scénario}

Deuxième dimension, les variables liées au scénario apparaissent conséquemment aux étapes choisies pour mettre en œuvre les transformations: le management participatif par la consultation régulière des acteurs, permet de conduire souplement ce changement et de réaliser des ajustements nombreux en cours de route. L'utilisation de l'encadrement comme animateur de la démarche est un des éléments significatifs du succès (Doutre \& Kouabenan, 1998). Enfin, le rythme, la planification ellemême est importante, elle doit tenir compte des variables contextuelles.

\section{Les acteurs du changement}

La direction (les dirigeants) tout d'abord a son importance, elle doit déterminer le contenu du projet et le légitimer. Elle doit aussi montrer l'exemple, c'est-à-dire s'impliquer dans les actions à changer. Les chefs de service ensuite, par leur fonction de relais de la direction, s'engagent en s'appropriant en premier les transformations.

\section{La progression du changement}

La mobilisation de la base, c'est-à-dire leur engagement organisationnel, employés et exécutants, n'est obtenue qu'en dernier lieu. On a constaté qu'ils s'approprieront d'autant plus les changements si préalablement l'encadrement a montré l'exemple. Le processus de changement doit donc être pensé avec diverses étapes progressives, de nature complémentaire, et dont l'efficacité apparaît au final, lors de l'implication des acteurs les moins gratifiés. Ces étapes suivent un processus descendant (top down) de la direction au personnel d'exécution via un programme composé de formation suivies d'actions permettant au projet de changement d'être pris en compte par l'ensemble du personnel afin de rendre ce dernier contributif des actions nouvelles à mettre en œuvre. On demandera au personnel d'exécution d'être force de proposition dans la concrétisation des différents projets. Et cette participation se fera de manière remontante (bottom up).

Diverses études (Rondeau, 1999) ont permis d'identifier une vingtaine de variables différentes présentées dans le tableau 2.

Tableau 2. Les variables significatives dans la conduite du changement

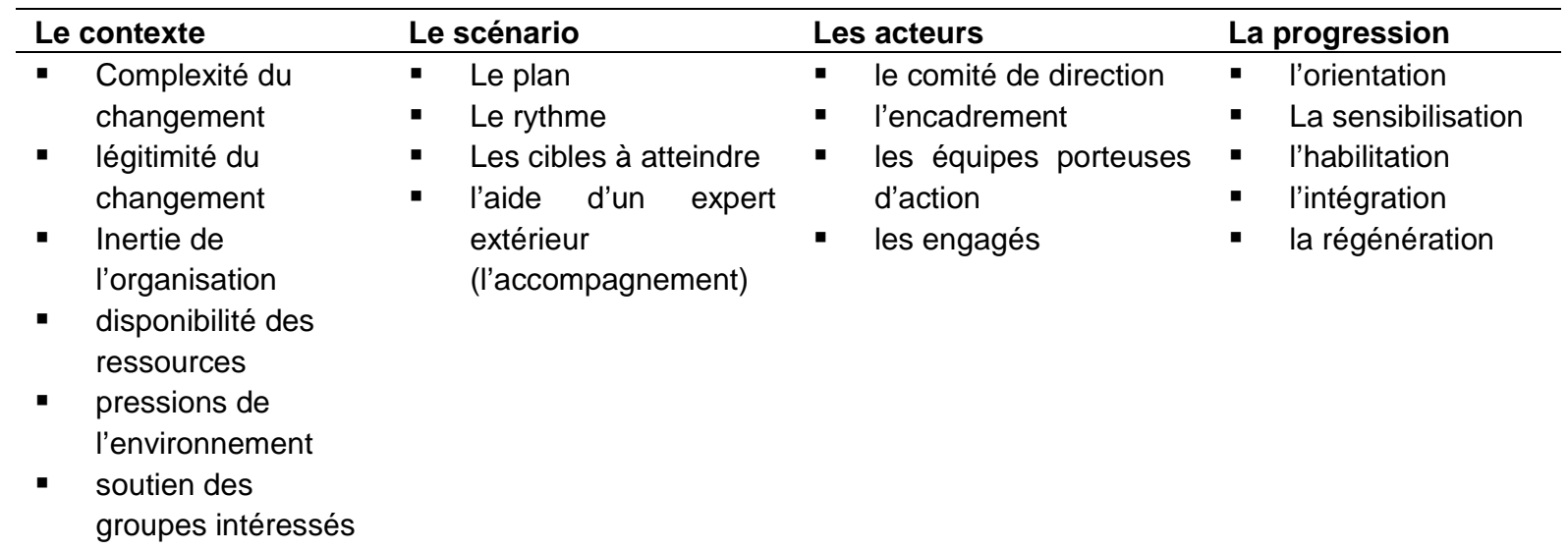

Changer, exige donc que l'organisation elle-même, ses systèmes, ses façons de faire et ses acteurs évoluent. On s'aperçoit que le développement de cette capacité se crée grâce à des activités qui vont permettre aux acteurs de comprendre la nature des changements visés et surtout qui vont leur permettre de s'engager dans les transformations.

Ces acteurs vont devoir développer de nouvelles compétences, s'adapter et ajuster leurs comportements aux nouveautés.

On comprend alors le lien d'intérêt qui existe entre la conduite du changement organisationnel et l'engagement 
organisationnel: ce sont des liens interactifs. L'engagement organisationnel impacte sur les succès du changement. On peut comprendre que la force, ou la réalité d'un engagement organisationnel est significatif dans l'évaluation d'une conduite de changement. II a été aussi montré que le changement provoque une mobilisation des acteurs qui vont s'engager dans des actions (Paillé, 2006; Neves, 2003; Huy, 1999). II y a donc des interdépendances entre toutes les variables en jeu, qu'elles soient d'engagement et/ou de changement. Cette imbrication explique pourquoi il est important de comprendre leurs mécanismes d'interaction et d'identifier les modèles en présence. Cependant, nous ne savons pas encore de façon consistante quel type d'engagement est le plus explicatif de la réussite d'un changement organisationnel. II y a là des perspectives réelles de recherche, notamment dans les organisations publiques où le type d'organisation bureaucratique peut renforcer les résistances des acteurs à changer.

\section{Le changement dans le service public: des particularités organisationnelles}

La question de la modernisation des services publics entamée depuis maintenant plusieurs années en France (Weller, 1998) a permis l'émergence de questionnement autour des problématiques d'efficience, de performance et de qualité de service. La perception naïve n'est pas toujours favorable vis-à-vis des fonctionnaires. On leur reproche un manque «d'engagement " (Warin, 2004). On peut le comprendre par une relecture des travaux de Weber $(1900,1917$, in écrits de 1957). C'est le premier à avoir analysé systématiquement le concept d'organisation. II a identifié deux forces primordiales qui la composent et qui s'opposent, la division du travail et la centralisation de l'autorité. La division du travail fragmente les organisations en les traduisant en éléments simples et de plus en plus spécialisés. A l'opposé, la centralisation de l'autorité va tendre à unifier et Weber parle de «coordination». Le but de la centralisation de l'autorité est de synchroniser les parties fractionnées de façon qu'elles fonctionnent en harmonie pour servir les objectifs de l'organisation.

Une des difficultés majeures est donc d'établir un équilibre entre ces forces: plus la spécialisation augmente, plus l'amélioration des techniques de coordination doit être plus grande. La rationalité à l'intérieur des organisations, débat des théoriciens et des organisateurs, repose sur l'effet réciproque des forces de spécialisation et de coordination. (Warin, 2004).

\section{Les principes qui régissent le Service Public}

Urwick (1937) a structuré les caractéristiques de la théorie bureaucratique de Weber en mettant en évidence les trois éléments essentiels, spécialisation, coordination et distribution rationnelle des tâches, et il établit que ses principes sont universels. C'est sur ces bases qu'aujourd'hui fonctionne le service public selon six principes fondamentaux:

un principe de continuité permettant la régularité de fonctionnement - par exemple, des horaires de travail, et des procédures délimitant les tâches à accomplir -. Ce principe de continuité ne peut avoir le même contenu pour tous les services, il existe donc une échelle de continuité selon la nature du service à rendre, le but de ce principe est d'obtenir une régularité de fonctionnement.

Un principe d'égalité, qui s'est développé à partir de la déclaration des droits de l'homme de 1789 (article 6) et qui conduit à la standardisation des services publics pour assurer un niveau égal de service pour tout le monde. II ne doit pas y avoir de différenciation de prix et de qualité entre chacun des services publics de même type.

- Un principe de mutualité: principe d'adaptation, les services publics sont appelés à évoluer en fonction des besoins et en fonction de l'appréciation de ces besoins par les autorités publiques. L'adaptation du service public consiste généralement en une amélioration du service par une simplification des procédures, une modification des conditions d'accès ou, à la limite, par une suppression du service. L'adaptation du service public est donc réalisée habituellement par voie unilatérale, les usagers ne pouvant pas se prévaloir d'un droit au maintien d'une réglementation déterminée à travers des accords avec les collectivités locales.

Un principe de transparence, les services publics doivent donner la possibilité aux usagers de s'informer sur l'activité de certains services, notamment sur l'utilisation des fonds financiers. Cependant, cette demande de transparence pour les usagers 
n'existe pas dans l'enseignement supérieur par exemple..

Un principe de spécialisation, englobant la division du travail et la spécialisation des tâches, les employés étant recrutés sur "concours " par rapport à des standards objectifs définis. Ce principe ayant pour but de "rationaliser» la gestion du personnel administratif.

Enfin, un principe de coordination par la centralisation de l'autorité afin d'établir un équilibre entre les différentes forces qui peuvent s'opposer.

\section{Les paradoxes}

On comprend l'origine d'un premier paradoxe dans la mise en perspective des conséquences de cette organisation du travail et les principes qui fondent le service public et lui donnent son essence, créant la culture bureaucratique considérée comme un modèle de qualité, voir comme «le » modèle pour ses agents (Warin, 2004): pourquoi changer puisque ce modèle est le meilleur !

Le deuxième paradoxe se trouve dans la logique floue du fonctionnement des services publics. Une des illustrations par exemple peut se trouver dans la mise en place de la réduction du temps de travail à 35 heures: alors que les organisations privées, particulièrement l'industrie, ont reconfiguré leurs processus pour gagner des postes, le public passe aux 35 heures sans avoir envisagé les conséquences de cette diminution des heures travaillées, laissant ainsi supposer, que les agents travaillant peu, peuvent se permettre une augmentation de productivité sans changement d'organisation du travail ni de comportement ! Cette conviction qui rejoint la pire des représentations du service public n'est pas sans conséquence sur la qualité du service rendu et la résistance des agents (François, 2003).

\section{Les conséquences du modèle public}

Le secteur public est rigide, il y a peu de mesure ni d'évaluation réelle car il est régi par une autorité lointaine et diffuse qui peut radicalement changer d'orientation puisqu'elle est politique et élue, et qui, pour assurer cette continuation se réfère à ses principes de fonctionnement immuables. II n'y a pas de problématique de jugement, mais seulement un contrôle de respect des procédures.

Cette absence d'évaluation est l'un des freins à la performance (Beauvois, 1994), et peut expliquer les difficultés rencontrées dans différents services publics lorsque des décisions d'amélioration sont prises: la question posée a toujours été de savoir sur quels référentiels se comparer, et non pas de se poser la question de sa performance propre, de sa capacité à se remettre en cause (Tarquinio \& Lochot, 2000).

\section{La difficile mise en cuvre du changement dans le service public: succès et échecs}

La notion de "service» dans le sens de "service rendu à " présente plusieurs spécificités comme l'intangibilité (il est immatériel et rarement spécifié, il est difficile à mesurer et, isoler son efficacité est complexe), la simultanéité de la production et de la consommation (le service n'est ni stockable ni transportable, il se consomme en même temps qu'il se produit, il est donc difficile à contrôler), l'hétérogénéité (il est rarement reproduit à l'identique, il est difficile à formater et à étalonner) (Argyris, 1995).

II est alors possible de dire que le «service public » est intangible, l'usager consomme le service en même temps qu'il se produit. Par contre, c'est un service homogène où cependant, on rencontrera aussi des suites de comportements difficilement maîtrisables et provoquant un vécu émotionnel fort (Warin, 2004).

Enfin le mode de gouvernance généralement peu participatif et délégatif ne favorise pas l'engagement des personnes.

Dans ce contexte, l'engagement organisationnel peut-il être un levier d'accompagnement du changement, peut-il être mis en jeu pour diminuer les forces de résistances que l'on vient d'évoquer?

\section{L'engagement organisationnel dans un contexte de changement: les différentes perspectives et le cas de l'hôpital public}

Les hôpitaux Français sont confrontés depuis quelques années à de multiples changements appelés réformes hospitalières (1996), démarche qualité (2002), mise en oeuvre des 35 heures (2003), mise en place des pôles de santé (2006)... II est normal et 
même nécessaire que les organisations changent. Cependant, en moins de dix ans, l'hôpital public a été le lieu de processus si répétitifs et rapides que l'on est en droit de se demander comment le personnel se sent toujours engagé envers lui, et quels pourraient en être les modèles les plus prédicteurs face à ces altérations de l'équilibre fonctionnel du système de travail: le changement est devenu à la fois «le décor, le sujet et l'objet de la pièce qui se joue dans les organisations hospitalières " (Ribau, Fraisse \& Bayad, 2003, p.2106). Ce mouvement continu demande donc de la part des acteurs une capacité à rester maître de soi, et ce d'autant plus que l'on sait qu'une des réactions des individus est de résister au changement (Angle \& Lawson, 1993; Argyris, 1995).

Engagée en 1996, la réforme hospitalière a quelque peu changé les rôles et missions des chefs de service de soins au patient en réduisant la dimension "hiérarchique » au profit de la dimension « d'animation ». L'introduction des démarches qualité (accréditation) va demander aux médecins d'être efficaces, c'est-à-dire de soigner au mieux le patient compte tenu de ses symptômes, mais aussi, d'être efficient, c'est-à-dire de soigner le patient au moindre coût. Ces exigences introduisent une notion de performance économique et de performance collective. Nous savons que cette performance peut se développer grâce à un engagement affectif envers l'organisation (Meyer \& al, 1993). Cependant, cette performance risque de se détériorer en situation de changement car l'influence de l'environnement sur les comportements reste essentielle, l'interaction entre facteurs personnels et environnementaux est déterminante (Bandura, 1993). Pour soutenir cette performance, et permettre aux personnes d'agir sur l'environnement afin de reconstruire des points de repère (Ribau \& al., 2003), des formations accompagnent l'introduction de ces démarches. Elles ont pour buts de favoriser un changement des attitudes afin de maintenir une double implication, celui du supérieur, le médecin chef de service, et celui des autres membres de l'équipe (cadre de santé, infirmières, aides soignantes) (Ribau \& al., 2003). Or, la littérature semble indiquer que les employés s'impliqueraient dans des relations d'échange avec l'organisation de manière distinctes de celles qu'ils entretiendraient avec leur supérieur (Meyer \& al., 2001, Stinglhamber \& al, 2002). Un contexte de changement amènerait-il d'autres processus d'engagement?

L'ordonnance 96 - 346 de 1996 fait obligation aux établissements de santé de procéder à une évaluation régulière de la satisfaction des patients. La réalisation de ce type d'évaluation, en général des questionnaires de satisfaction, est censée susciter ou renforcer l'implication des équipes médicales dans une démarche qualité. La diffusion des résultats de ces questionnaires devrait avoir pour effet la mise en place d'actions correctives visant par voie de conséquence l'amélioration de la satisfaction des patients. Or, une revue de littérature notamment en la matière ne constate pas d'effet significatif de ces nouvelles pratiques (Parker \& Kroboth, 1991; Dull, Lansky \& Davis, 1994; Scott \& Smith, 1994; Tasa, Baker \& Murray, 1996; Rogers \& Smith, 1999; Cohen \& Buchan, 2001; Greco \& al, 2001; Ribau \& al, 2003). Une étude exploratoire récente sur cette question a permis de mettre en évidence un lien entre des variables personnologiques et comportementales des chefs de services et leur implication dans ces démarches qualité considérées comme introductives de changement. Deux variables ont été isolées: le style de leadership et le "Locus of Control » (ou degré de contrôle) (François \& Doutre, 2004). II semblerait qu'un style de leadership soit plus particulièrement prédicteur d'engagement des collaborateurs: le style transformationnel crée un terrain favorable pour la diffusion et l'utilisation de ces enquêtes. Ce résultat confirmerait ce que l'on savait par ailleurs: le style transformationnel serait le plus adéquat pour obtenir une motivation et une mobilisation du personnel comparé aux deux autres styles, le transactionnel et le passif évitant (Hetland \& Sandal, 2003, Bass, 1998, Bass, \& Steidlmeier, 1999, Barling, Slater, \& Kelloway, 2000). Concernant la deuxième variable, le LOC interne serait prédictible d'un passage aux actes, et s'inscrirait dans ce qui a été déjà mis en évidence par des études sur l'engagement interne aux sujets (Rotter, 1966, Pansu, 1997, Tafani \& Souchet, 2001, Spector $\&$ al, 2002). Les liens de ces deux variables avec I'EO ne sont pas nouveaux, mais n'ont pas fait l'objet d'une publication consistante (Meyer \& al, 2001, Meyer \& al, 2002). Cependant ces deux variables, combinées, n'ont pas encore été étudiées dans leurs effets sur un EO. Concernant l'engagement envers le supérieur pour les membres de l'équipe de 
soins, de nombreuses études sur l'hôpital ont également souligné l'importance de la collaboration infirmières/médecins pour l'obtention de résultats positifs en terme de performance - réduction du taux de décès, de la durée du séjour, du turnover infirmier et amélioration de la capacité des infirmières à répondre aux besoins des patients et de leurs familles (Knaus \& al, 1986, Mitchell \& al, 1989, Stordeur \& al, 2003). Ces travaux confortent l'hypothèse de l'influence de la personnalité et du comportement du chef médecin dans leur double effet sur son Engagement Organisationnel et sur celui de ses collaborateurs (I'EO des collaborateurs ne se développant pas vers l'organisation mais envers leur supérieur) dans un contexte de changement.

\section{L'importance des variables individuelles comme variables antécédentes à l'engagement organisationnel dans un contexte de changement organisationnel de service public}

La littérature suppose qu'un leader efficace, celui qui influence ses collaborateurs, s'adapte à son environnement, sache négocier leadership transactionnel (Bass, 1985)-, et cherche à le transformer - leadership transformationnel (Bass, 1999) - cette vision systémique du leadership s'intéresse à son action en deux temps sur l'équipe de travail, et propose un modèle managérial. Le premier temps met en adéquation le style de leadership - les conduites du chef vis-à-vis de ses collaborateurs, leurs compétences acquises et leur niveau de confiance en l'organisation. Cette adéquation situationnelle s'entend dans un contexte stable où des transactions sont possibles entre les objectifs à atteindre et les renforcements obtenus. Cependant, à long terme, cette première dimension du leadership ne semble plus prédictible de réussite faute de provoquer chez les collaborateurs des changements d'ordre supérieur comme les attitudes, les croyances et les valeurs. Aussi, le leader doit transformer l'environnement de ses collaborateurs en leur donnant du pouvoir de façon à ce qu'ils deviennent des agents de changement dans l'organisation. Ce style de leadership va s'appuyer sur le partage des actions qui engagera les deux parties, le chef et les collaborateurs. Ce leader transformationnel développe des qualités charismatiques, il sait parler et enthousiasmer, il pratique la considération individuelle, et il provoque la stimulation intellectuelle. Ce type de leader voit se développer dans son équipe de nombreuses innovations et de l'implication (Bass \& al, 1991; 1998; 1999). On peut légitimement valider le choix de cette variable dans un contexte de changement organisationnel.

\section{La combinaison des variables}

Le comportement du chef, que l'on appellera style de Leadership, permettrait alors de créer les conditions favorables dans un groupe à s'engager vis-à-vis du supérieur. Et ceci, notamment dans un contexte de changement parce qu'alors, le chef permettrait, par son comportement, de compenser la diminution de la relation identitaire de ses collaborateurs envers l'organisation. Parallèlement, le LOC serait prédictible d'engagement personnel à passer aux actes. Nous pensons que ces deux variables combinées peuvent être antécédentes de l'engagement organisationnel. La variable leadership ayant un effet prédictible d'influence sur le groupe et la variable LOC étant prédictible d'engagement personnel, il serait pertinent de les lier afin de repérer si elles sont prédictibles l'une vis-à-vis de l'autre et si elles peuvent avoir le double effet attendu: sur le chef et sur ses collaborateurs en contexte de changement.

\section{Perspectives de recherche}

Si l'implication dans la démarche qualité (ayant pour effet, dans un contexte de changement, d'exploiter des questionnaires de satisfaction des patients) est attendu de la part des médecins chefs de service (variable dépendante), peut-on admettre que plus un chef sera interne plus on obtiendra cette implication (Beauvois, 1994, Doutre, 2003)? Par ailleurs, l'engagement organisationnel dans sa dimension affective (EA) attendu de la part des médecins chefs de service pour exercer leurs rôles, permet d'espérer qu'un chef interne peut avoir un sentiment d'appartenance à l'organisation (EA) plus important qu'un externe, et qu' il s'impliquera plus. Cette implication l'amènerait à exploiter les questionnaires de satisfaction des patients.

Par ailleurs, on peut aussi admettre qu'un chef interne saurait pratiquer la reconnaissance des investissements personnels de ses collaborateurs et, par conséquent, leur permettrait de s'attacher à lui et de développer 
de la loyauté. Le LOC interne du chef pourrait avoir alors une antécédence sur l'engagement affectif de ses collaborateurs via la pratique de la reconnaissance et le développement de la loyauté. Cet EA envers le supérieur permettrait alors aux collaborateurs de s'impliquer, en participant à des actions correctives. Le LOC aurait donc une influence sur les deux types d'engagement, organisationnel et envers le supérieur dans un contexte de changement.

Enfin, le lien entre un LOC interne et un style de leadership transformationnel paraîtrait alors prédictible de conduites "responsables » d'un chef qui débattra des problèmes rencontrés, (le contenu des questionnaires de satisfaction des patients), et prendra une décision de manière concertée avec ses collaborateurs en les amenant à réaliser des actions correctives. On peut donc supposer que les collaborateurs engagés affectivement envers leur chef accepteront de réaliser ces actions correctives.

\section{Conclusion}

L'engagement affectif des salariés est il un moyen de contourner la résistance au changement? Tout dépend du contexte environnemental et des typologies d'acteurs en présence: nous avons vu que l'EA semble permettre de contourner cette résistance. Cependant, ces connaissances restent à vérifier dans le domaine de l'organisation publique et notamment à l'hôpital. Dans ce travail, nous avons tenté de comprendre comment s'organisent et se structurent les mécanismes d'engagement organisationnel. Nous avons pu mettre en évidence l'intérêt d'utiliser le type d'engagement effectif envers le supérieur comme variable ayant un effet sur la diminution de la résistance au changement. Cette variable est pertinente car si elle se rencontre dans une situation de travail concomitamment à l'existence d'un chef transformationnel et interne, on obtiendra, peut être, un engagement des collaborateurs dans l'action. Cet engagement dans l'action peut être entendu comme la preuve d'une diminution de la résistance au changement.

La résistance au changement est certes contournable autrement. Les différents programmes mis en œuvre ici ou là (Rondeau, 1999) montrent cependant les difficultés rencontrées, notamment en matière de temps. En effet, le temps pour obtenir une diminution de la résistance au changement est long, demande très souvent de l'accompagnement (Rondeau, 2002; Paillé, 2006). La perspective d'obtenir un engagement organisationnel, dans les conditions que nous supposons, peut vraisemblablement aussi jouer sur la variable temps: la résistance au changement pourrait diminuer de manière plus significative et plus rapidement. C'est une hypothèse que cette revue de question rend légitime.

Enfin, ce travail prend un intérêt supplémentaire lorsque l'on s'intéresse au service public: malgré ses paradoxes et sa rigidité, la diminution de la résistance au changement pourrait aussi s'obtenir dans un contexte d'engagement organisationnel.

\section{Résumé}

Ce travail a pour but de repérer quelles sont les variables organisationnelles mises en jeu en contexte de changement et comment elles s'organisent et s'articulent entre elles. Notamment lorsqu'elles jouent conjointement sur l'engagement organisationnel du chef et de ses collaborateurs et ceci dans le contexte spécifique du service public Français. Le cas de l'hôpital public servira à identifier quelques perspectives de recherches et d'application.

Mots clés: Engagement organisationnel, engagement affectif, engagement envers le supérieur, changement organisationnel, service public.

\section{Références}

Allen, N.J., \& Meyer, J.P. (1990). The measurement and antecedents of affective, continuance and normative commitment to the organization. Journal of occupational Psychology, 63, 1-18.

Allen, N.J., \& Meyer, J.P. (1991). A three component conceptualization of organiszational commitment. Human Resource Management Review, 1,1, 61-89.

Allen, N.J., \& Meyer, J.P. (1996). Affective, continuance and normative commitment to the organization: an examination of construct validity. Journal of Vocational Behavior, 49, 252-276.

Angle, H.L., \& Perry, J.L. (1983). Organzational commitment: individual and organizational influences. Work and Occupational, 10, 2, 123-146.

Angle, H.L., \& Lawson, M.B. (1993). Changes in affective and continuance commitment in 
times of relocation. Journal of Business Research, 26, 1, 3-15.

Argyris, C. (1995). Savoir pour agir, surmonter les obstacles à l'apprentissage organisationnel. Paris: InterEditions.

Bandura, A. (1993). Perceived self-efficacy in cognitive development and functioning. Educational Psychology, 28: 117-148.

Bandura, A. (2001). Social cognitive theory: An agentic perspective. Annual Review of Psychology, 52, 1-26.

Barling, J. Slater, F., \& Kelloway, E.K. (2000). Transformational leadership and emotional intelligence: an exploratory study. Leadership and Organizational Development Journal, 21, 157-161

Bass, B.M. (1985). Leadership and performances beyond expectations. New York: Free Press.

Bass, B.M. \& Avolio, B.J. (1991). Multifactor Leadership Questionnaire (Form 5X), Center for leadership studies. School of Management, Binghamton University.

Bass, B.M. (1998). Transformational leadership: Industrial, military, and educational impact. New York. Hillsdale, N.J, Erlbaum.

Bass, B.M. \& Steidlmeier, P. (1999). Ethics, charater, and authentic transformational leadership behavior. Leadership Quaterly, 10, 181-217.

Beauvois, J.L.\& Joulé, R.V. (1985). Soumission et idéologies, Psychosociologie de la rationalisation. Paris: Presses Universitaires de France.

Beauvois, J.L. (1994). Traité de la servitude libérale: analyse de la soumission. Paris: Dunod.

Becker, H.S. (1960). Notes on the concept of commitment. American Journal of Sociology, $66,1,32-42$.

Blau, G. (1999). Testing the generalizability of a career commitment measure and its impact on employee's turnover. Journal of Vocational Behavior, 1, 331-343.

Brasseur, M., \& Mzabi, H.J. ( 2003). L'implication des salariés, clef de réussite du changement. GRH, Innovons, 1, 437-462. Paris: AGRH Ed.

Brown, R.B. (1996). Organizational commitment: clarifying the concept and simplifying the existing construct typology. Journal of Vocational Behavior, 49, 230-251.

Cohen, A. (2000). The relationship between commitment forms and works outcomes: a comparison of three models. Human Relations, 53, 3, 387-417.

Cohen, G., \& Buchan, J. (2001). Can different patient satisfaction survey methods yield consistent results? comparison of three surveys. British Medical Journal, 313, 841844

Commeiras, N. (1994). L'intéressant facteur d'implication organisationnelle: une étude empirique. Thése de Doctorat en sciences de gestion, IAE, Montpellier.

Cornet, A. (1999). Dix ans de réingénierie des processus d'affaires: qu'avons-nous appris? Gestion, 24, 3, 66-75.

DeCotiis T.A., \& Summers, T.P. (1987). A parth analysis of a model of the antecedents and consequences of organizational commitment. Human Relations, 40, 7, 445-470.

Delobbe, N., \& Vandenberghe, C. (2000). A four dimensional model of organizational commitment among Belgian employees. European Journal of Psychological Assessment, 16, 2, 125-138.

Doutre, E. (2003). Améliorer l'investissement personnel: lien entre pratique du bilan de compétence et internalité, in N. Delobbe, G. karnas, \& C. Vandenberghe, (Eds), Evaluation et développement des compétences au travail. Louvain: PUL, 307319.

Doutre, E., \& Kouabenan, D.R. (1998). Engagement librement consentie et démarche qualité, Communication présentée au 5ième congrès international de langue française, ADRIPS, Turin les 28, 29 et 30 Août.

Dull, VT., Lansky, D., \& Davis, N. (1994). Evaluating a patient satisfaction survey for maximum benefit. The Joint Commission Journal on Quality Improvement, 20, 8, 444-453.

Durrieu, F., \& Roussel, P. (2002). L'implication organisationnelle dans les reseaux de franchise: un concept pour les enterprises en réseau, Revue de Gestion des Ressources Humaines, 44, 2-19

Eagly, A., \& Chaiken, S. (1993). The Psychology of attitudes. New York: Harcourt Brace Jovanovich.

Eisenberger, R. Stinglhamber, F. Vandernberghe, C. Sucharski, I., \& Rhoades, L. (2002). Perceived supervisor support: contributions to perceived organizational support and employee retention. Journal of Applied Psychology, 87, 565-573.

El Akremi, A., \& Trabelsi, Y. (2003). Rôle des pratiques de $\mathrm{GRH}$ dans le développement de l'engagement organisationnel des travailleurs intellectuels. GRH, Innovons, 2, 1031 - 1063. Paris: GRH Ed.

Festinger, L. (1957). A theory of cognitive dissonance, Stanford University Press.

Fiedler, F.E. (1986). The contribution of cognitive resources of leadership performance. In L. 
Berkowitz (Ed.), Advances in Experimental Social Psychology. NY: Academic Press.

Fisch, R. Weakland, J.H., \& Segal, L. (1983). The tactics of change. San Francisco: JosseyBass,

Fisbein, M., \& Ajzen, L. (1975). Belief, Attitude, Intention, and Behavior. Reading: AddisonWesley.

François, P. (2003). La démarche qualité à l'hôpital. $4^{\text {ème }}$ Colloque du Greypsit Rhône Alpes, «Management de la qualité: état des recherches en sciences sociales ». Grenoble le 23 janvier.

François, P., \& Doutre, E. (2004). Perception et utilisation des résultants d'enquête de satisfaction des patients par les professionnels des services cliniques d'un hôpital. Revue Risques et Qualité en Milieu de Soin, Janvier, 1, 15-21.

Gaertner, K.N., \& Nollen S.D. (1992). Turnover intentions and desire among executives. Human Relations, 45, 5, 447-465.

Greco, M. Brownlea, A., \& Mc Govern, J. (2001). Impact of patient feedback on the interpersonal skills of general practice registrars: results of a longitudinal study. Medical Education, 35, 8, 748-756

Herrbach, O., \& Mignonac, K. (2002). Une étude qualitative des cibles et formes d'engagement et de leurs liens avec la performance individuelle. Psychologie $d u$ Travail et des Organisations, 8, 3, 39-68.

Hersey, P., \& Blanchard, KH. (1987). Management of organizational behaviour, utilizing human resources. Englewood Cliffs: Prentice Hall.

Hetland, H., \& Sandal, G.M. (2003). Transformational Leadership in Norway: outcomes and personality correlates. European Journal of Work and Organizational Psychology, 12, 2, 147-170.

Huy, Q. (1999). Emotional capability, emocioal intelligence and radical change. Academy of Management Review, 24, 2, 325-345.

Joulé, R.V., \& Beauvois, J.L. (1984). Petit traité de manipulation à l'usage des honnêtes gens. Paris: PUG

Judson, A. (1991). Changing behavior in organizations: minimising resistance to change, Cambridge: Bazil Blackwell.

Kanter, R.M. (1968). Commitment and social organization: a study of commitment mechanisms in utopian communities. American Sociological Review, 33, 4, 499517.

Knauss, W.A., Draper, E.A., Wagner, D.P., \& Zimmerman, J.E. (1986). An evaluation of outcome from intensive care in major medical centers. Annals of Internal Medicine, 104, 410-418.

Kotter, J.P. (1996). Leading change. Boston: Harvard Business School Press.

Lincoln, J.R., \& Kallenberg, A.L. (1996). Commitment, quits, and work organization in Japanese and US plants. Indutrial, Labor Relations Review, 50, 1, 39-60.

Lewin, K. (1951). Field Theory in social science. New York: Harper Collins

Mathieu, J.E., \& Zajac, D.M. (1990). A review and meta-analysis of the antecedents, correlates and consequences of organizational commitment. Psychological Bulletin, 108, 2, 171-194.

Mannheim, B. Baruch, Y., \& Tal, J. (1997). Alternative models for antecedents and outcomes of work centrality and job satisfaction of high-tech personnel. Human Relations, 50, 12, 1537-1562.

May T.Y.M., Korczynski, M., \& Frenkel, S.J. (2002). Organizational and occupational commitment: knowledge workers in large corporations, Journal of Management Studies, 39, 6, 775-801.

Meyer, J.P., Allen, N.J., \& Smith, C.A. (1993). Commitment to organization and occupations: extension and test of threecomponent conceptualization. Journal of Applied Psychology, 78, 4, 538-551.

Meyer, J.P., \& Allen, N.J. (1997). The commitment in the workplace: Theory, research and application. Sage Publications.

Meyer, J.P., \& Smith, C.A. (2000). HRM Practices and organizational commitment: test of a mediation model. Canadian Journal of Administrative Sciences, 17, 4, 319-331.

Meyer, J.P., \& Hersovitch, L. (2001). Commitment in the workplace: toward a general model. Human Ressource Management Review, 11, 299-326.

Meyer, J.P., Stanley, D.J., Hercovitch, L., \& Topolnytsky, L. (2002). Affective, continuance and normative commitment to the organization: a meat-analysis of antecedents, correlates and consequences. Journal of Vocational Behavior, 61, 20-52.

Mintzberg, H. Ahlstrand, B. \& Lampel, J. (1999). Transformer l'entreprise. Gestion, 24, 3, 148-157.

Mitchell, P.H., Armstrong, S., Simpson, T.F., \& Lentz, M. (1989). American Association of critical-nurses demonstration project: profile of excellence in critical care nursing. Heart and Lung, 18, 3, 219-239.

Morrow, P. C. (1983). Concept redundancy in organizational research: the case of work 
commitment. Academy of Management Review, 8, 486-500.

Mowday, R.T., Porter, L.W., \& Steers, R.M. (1982). Employee-organization linkages: the psychology of commitment, absenteeism and turnover. New York: Academic Press.

Neves J. (2003). Changer la culture organisationnelle, in C. Vandenberghe, $\mathrm{N}$. Delobbe, \& G. Karnas, (Eds), Dimensions individuelles et sociales de l'investissement professionnel. Louvain: PUL, 435-448.

O'Reilly, III, C., \& Chatmann, J. (1986). Organizational commitment and psychological attachment: the effect of compliance, identification and internalisation on prosocial behaviour. Journal of Applied psychology, 73, 3, 492-499.

Paillé, P. (2006). Contribution à l'étude du concept d'implication organisationnelle, analyse des expériences d'un changement et mise en perspective du rôle de l'engagement dans l'emploi, Thèse de Doctorat en Sciences de gestion, Université de Toulouse.

Palmero, F. (2000). Implication organisationnelle, satisfaction au travail, engagement au travail et intention de départ des salariés à temps partiel, communication, $11^{e}$ congrès de l'AGRH, ESCP.

Pansu, P. (1997). Norme d'internalité et appreciation de la valeur professionnelle: l'effet des explications internes dans l'appréciation du personne. Le travail Humain, 60, 2, 205-222

Parker, SC., \& Kroboth, FJ. (1991). Practical problems of conducting patient-satisfaction survey. Journal of General Internal Medecine, 5, 430-435.

Reichers, A.E. (1985). A review and reconceptualization of organizational commitment. Journal of Vocational Behavior, 36, 210-224.

Ribau, N., Fraisse, S., \& Bayad, M. (2003). La nécessité de repenser l'accompagnement du changement dans les hôpitaux Français. $\mathrm{GRH}$, Innovons, $14^{\mathrm{e}}$ congrés de l'AGRH, Grenoble, 3, 2103-2127.

Rogers, G., \& Smith, D. (1999). Reporting comparative results from hospital patient surveys. International Journal of Quality Health Care, 5, 51-54

Rondeau, A. (1999). Transformer l'organisation, vers un modèle de mise en œuvre. Gestion, 24, 3, 148-157.

Rondeau, A.. (2003). Suivi et diagnostic d'une restructuration, in N. Delobbe, G. karnas, \& C. Vandenberghe, (Eds). Bien-être au travail et transformations des organisations, 583-591.

Rotter, JB. (1966). Generalized expectancies for internal versus external control of reinforcement. Psychological Monographs, 80, 609-621

Rosenberg, M.J., \& Howland, C.I. (1960). Cognitive, affective and behavioral components of attitudes, in Holland, C.I. \& Rosenberg, M.J., Attitude organizational and change: an analysis of consistency among attitude components. New Haven, CT: Yale University Press.

Simard, G. (2000). Les antécédents de l'engagement organisationnel: le cas d'une organisation publique du secteur de la santé, Communication 196, 11e congrès de L'AGRH, Paris.

Scott, A., \& Smith, RD (1994). Keeping the customer satisfied: issues in the interpretation and use of patient satisfaction surveys. International Journal for Quality in Health Care, 6, 4, 353-359.

Spector, P.E., Cooper, C., \& Aguilar-Vafaie, M.E. (2002). A comparative study of perceived job stressor sources and job strain in American and Iranian Managers. Applied Psychology, and International Review, 51, 3, 446-457.

Stinglhamber, F. Bentein, K., \& Vandenberghe, C. (2002). Extension of the tree component model of commitment to five foci: development of measures and substantive test. European Journal of Psychological Assesment, 18, 123-138.

Stordeur, S., Vandenberghe, C., \& D'Hoore, W. (2003). Leadership transformationnel des infirmières et contexte de travail, in Vandenberghe, C, Delobbe, N. \& Karnas, G. (Eds.) Dimensions individuelles et sociales de l'investissement professionnel. Louvain, PUL, 345-376.

Tafani, E., \& Souchet, L. (2001). Changement d'attitude et dynamique représentationnelle, in Moliner, P., La dynamique des représentations sociales. Chap. 3, PUG, collection « vies sociales ».

Tajfel H., Bilig M., Bundy R.P., \& Flament, C. (1999). Social catégorisation and intergroup behavbiour, European Journal of Social Psychology, 1, 149-178 cité et traduit par Geneviève Vinsonneau. Inégalités sociales et procédés identitaires, Paris: Armand colin.

Tarquinio, C., \& Lochot, F. (2000). Les conditions d'une ingénierie psychosociale réussie: réflexions à partir d'une démarche d'assurance qualité en formation. Actes du 
3iéme Congrès International de Psychologie Sociale en Langue Française, 2, 325 - 335.

Tasa, K. Baker, R., \& Murray, M. (1996). Using patient feedback for quality improvement. Quality Management in Health Care, 4, 55-67

Thevenet, M. (1993). L'implication au travail, Les cahiers de l'ANVIE. Paris: Sciences Humaines, 28, 32-45

Urwick, L. (1937). Papers in the Science of Administration. Institute of Public Administration.

Warin, Ph. (2004). Quelle modernisation des services publics? Les usagers au cœur de la réforme. Paris: La découverte

Varona, F. (1996). Relationship between communication satisfaction and organizational commitment in three Guatemalan organzations. The Journal of Business Communication, 33, 2, 111-140.
Watzlawick, P., Weakland, J., \& Fisch, R. (1975). Changement, Paradoxes et Psychothérapies. Paris: Seuil.

Weber, M, (1952). The essentials of bureaucratic Organisation: an ideal-type construction. K. Robert; L. Merton (Eds). Reader in Bureaucracy. New York: The Free Press.

Weller, J.M. (1998). La modernisation des services publics par l'usager: une revue de la littérature 1986-1996. Sociologie du Travail, 3, 365-392.

Wiener, Y., \& Vardi, Y. (1980). Relationship between job, organization, and work outcomes: subgroup hypothesis revisited. Organizational Behavior and Human Performance, 26, 81-86.

Yoon, J. Baker, M.R., \& Ko, J.W. (1994). Interpersonal attachment and organizational commitment; Evidence for differences among multileved attachments. Human Relations, 47,3, 329-351.

\section{D\&D Consultants, Bucureşti \\ www.ddconsultants.ro}

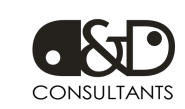

\section{Instrumente psihometrice publicate de D\&D Consultants/TestCentral}

CPITM (California Psychological Inventory $(462,434,260)$ )

NPQTM (Nonverbal Personality Questionnaire)

FFNPQTM (Five-Factor Nonverbal Personality Questionnaire)

SWS ${ }^{\mathrm{TM}}$ (Survey of Work Styles)

STAXI-2 ${ }^{\text {TM }}$ (State-Trait Anger Expression Inventory)

FPITM (Freiburg Personlichkeitsinventar (Formele G şi R))

$\mathrm{LSI}^{\mathrm{TM}}$ (Learning Styles Inventory)

MLQTM (Multifactor Leadership Questionnaire (Forma 5X))

STAITM (State-Trait Anxiety Inventory)

STAICTM (State-Trait Anxiety Inventory for Children)

JVIS ${ }^{T M}$ (Jackson Vocational Interest Survey)

AMI $^{\text {TM }}$ (Achievement Motivation Inventory)

FJASTM (Fleishman Job Analysis Survey)

Instrumente psihometrice în curs de apariție:

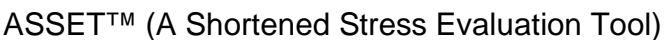

EPQTM-R (Eysenck Personality Questionnaire, Revised)

IVETM (Eysenck's Impulsiveness Questionnaire)

BFQ $^{T M}$-2 (Big Five Questionnaire)

BFA $^{\mathrm{TM}}$ (Big Five Adjectives)

GAMA $^{\text {TM }}$ (General Ability Measure for Adults)

$\mathrm{MAB}^{\mathrm{TM}}$-II (Multidimensional Aptitude Battery)

NEO-PI-R ${ }^{\mathrm{TM}}$ (Revised NEO Personality Inventory)

BASC-2 ${ }^{\mathrm{TM}}$ (Behaviour Assessment System for Children, Second Edition)

MSCEITTM (Mayer Salovey Caruso Emotional Intelligence Test)

EO-ITM (Emotional Quotient Inventory)

TEDE6 ${ }^{\mathrm{TM}}$ (Test d'évaluation dynamique de l'éducabilité)

DDDITM (Dula Dangerous Driving Index) 